\title{
Comment on:"Prevalence and distribution of human papillomavirus genotypes in cervical intraepithelial neoplasia in China: a meta-analysis"
}

\author{
Hongxi Zhang ${ }^{1} \cdot$ Mi Tang $^{2} \cdot$ Yongxian Jiang $^{3} \cdot$ Jianghui Cai $^{1}$ (D) \\ Received: 3 February 2021 / Accepted: 24 January 2022 / Published online: 1 February 2022 \\ (c) The Author(s), under exclusive licence to Springer-Verlag GmbH Germany, part of Springer Nature 2022
}

\section{Dear Editor,}

We read with great interest the article by Zhang et al. (volume 302, issue 6) [1]. The authors summarized and demonstrated the prevalence and distribution of human papillomavirus (HPV) genotypes in cervical intraepithelial neoplasia (CIN). Certainly, the findings of Zhang et al. hold significance for future HPV-based cervical cancer screening tests, treatment of HPV infection, and application of HPV vaccines in China. However, we raise several concerns regarding this systematic review and meta-analysis.

First, only Medline, Embase, and Cochrane Library databases were searched for potential articles, which may result in reporting bias. This study aimed to investigate the prevalence and distribution of HPV genotypes between January 2000 and April 2019 in China. We think that it is appropriate to perform a comprehensive literature search of the Chinese Biomedical Literature database (regional database) to identify as many eligible studies published in Chinese as possible.

Second, we cannot find the PRISMA checklist and registration number (Cochrane or PROSPERO) in the manuscript or supplementary files.

Jianghui Cai

776773221@qq.com

1 Department of Pharmacy, Chengdu Women's and Children's Central Hospital, School of Medicine, University of Electronic Science and Technology of China, No. 1314 Riyue Avenue, Qingyang District, Chengdu 610041, China

2 Office of Good Clinical Practice, Chengdu Women's and Children's Central Hospital, School of Medicine, University of Electronic Science and Technology of China, Chengdu 610041, China

3 Department of Pharmacy, Sichuan Provincial Maternity and Child Health Care Hospital, Chengdu Sichuan, 610041, China
Third, the authors should present a full electronic search strategy for at least one database, including any limits used, such that it could be repeated [2].

Fourth, the authors stated 'the data from all the studies were collected and checked simultaneously by the researchers' in the Data abstraction section. However, in the Author Contributions section, only one author had roles in the data collection and analysis. Selection of studies or data extraction was made by one independent investigator, which is inadequate.

Five, the authors did not assess the risk of bias in individual studies according to the PRISMA recommendations, which are mandatory for any systematic review.

Author contributions HZ: manuscript writing. MT: manuscript writing. YJ: manuscript writing. JC: project development. All authors reviewed, contributed to the work equally and approved the final version.

Funding None.

\section{Declarations}

Conflict of interest We declare no competing interests.

Ethical approval Not applicable.

\section{References}

1. Zhang J, Cheng K, Wang Z (2020) Prevalence and distribution of human papillomavirus genotypes in cervical intraepithelial neoplasia in China: a meta-analysis. Arch Gynecol Obstet 302:1329-1337

2. Moher D, Liberati A, Tetzlaff J et al (2009) Preferred reporting items for systematic reviews and meta-analyses: the PRISMA statement. BMJ 339:b2535

Publisher's Note Springer Nature remains neutral with regard to jurisdictional claims in published maps and institutional affiliations. 